\title{
Genetic parameters of linear conformation type traits and their relationship with milk yield throughout lactation in mixed-breed dairy goats
}

\author{
A. McLaren, ${ }^{*}$ S. Mucha,$\dagger^{1}$ R. Mrode, $† \ddagger$ M. Coffey, $\dagger$ and J. Conington $\dagger$ \\ *Scotland's Rural College, Hill and Mountain Research Centre, Kirkton Farm, Crianlarich, FK20 8RU, United Kingdom \\ †Scotland's Rural College, Animal and Veterinary Sciences, Easter Bush, Midlothian, EH25 9RG, United Kingdom \\ ¥Animal Biosciences, International Livestock Institute, PO Box 30709, Nairobi 00100, Kenya
}

\begin{abstract}
Conformation traits are of interest to many dairy goat breeders not only as descriptive traits in their own right, but also because of their influence on production, longevity, and profitability. If these traits are to be considered for inclusion in future dairy goat breeding programs, relationships between them and production traits such as milk yield must be considered. With the increased use of regression models to estimate genetic parameters, an opportunity now exists to investigate correlations between conformation traits and milk yield throughout lactation in more detail. The aims of this study were therefore to (1) estimate genetic parameters for conformation traits in a population of crossbred dairy goats, (2) estimate correlations between all conformation traits, and (3) assess the relationship between conformation traits and milk yield throughout lactation. No information on milk composition was available. Data were collected from goats based on 2 commercial goat farms during August and September in 2013 and 2014. Ten conformation traits, relating to udder, teat, leg, and feet characteristics, were scored on a linear scale (1-9). The overall data set comprised data available for 4,229 goats, all in their first lactation. The population of goats used in the study was created using random crossings between 3 breeds: British Alpine, Saanen, and Toggenburg. In each generation, the best performing animals were selected for breeding, leading to the formation of a synthetic breed. The pedigree file used in the analyses contained sire and dam information for a total of 30,139 individuals. The models fitted relevant fixed and random effects. Heritability estimates for the conformation traits were low to moderate, ranging from 0.02 to 0.38 . A range of positive and negative phenotypic and genetic correlations
\end{abstract}

Received August 17, 2015.

Accepted March 4, 2016.

${ }^{1}$ Corresponding author: Sebastian.Mucha@sruc.ac.uk between the traits were observed, with the highest correlations found between udder depth and udder attachment (0.78), teat angle and teat placement (0.70), and back legs and back feet (0.64). The genetic correlations estimated between conformation traits and milk yield across the first lactation demonstrated changes during this period. The majority of correlations estimated between milk yield and the udder and teat traits were negative. Therefore, future breeding programs would benefit from including these traits to ensure that selection for increased productivity is not accompanied by any unwanted change in functional fitness.

Key words: dairy goat, conformation, milk yield, random regression

\section{INTRODUCTION}

Conformation traits are of interest to many animal breeders, not only as descriptive traits in their own right but also because of their influence on production, longevity, and profitability (Brotherstone 1994). In dairy cattle, linear type traits were first recorded in the early 1980s (Meyer et al., 1987; Short and Lawlor, 1992; Brotherstone, 1994). In terms of small ruminants, one of the earliest scales was developed by the American Dairy Goat Association (ADGA), which has been used to score goats across a range of different breeds since 1988 (Luo et al., 1997). The linear traits included in this scale are scored from 1 to 50, with an additional score, based on overall appearance according to several weighted criteria, of between 50 and 99. However, several other scales, which have also been used to assess conformation traits, have been developed based on 9-point linear type scoring systems. One of the earliest proposed for dairy sheep was by de la Fuente et al. (1996) during an evaluation of Churra ewes, from which several other scales, adapted for different breeds, have emerged (Carta et al., 2009). In terms of dairy goats, a 9-point scale has been developed and is currently used by French dairy goat breeders, as described by Manfredi et al. (2001). 
Initially, the most commonly recorded traits were udder and teat type traits, mainly due to their influence on milking ability, udder health, and longevity of animals. More recently, several studies have considered additional traits, such as those relating to the legs and feet of the animals (Manfredi et al., 2001; de la Fuente et al., 2011). By improving aspects such as animal mobility and structural correctness, it is possible that productivity and profitability of dairy goat herds could further improve, particularly when coincidentally exposed to intense selection pressure for yield traits.

If conformation traits are to be considered for inclusion in future dairy goat breeding programs, relationships between the conformation traits themselves, as well as with production traits such as milk yield, must be considered. Fernandez et al. (1997) and Legarra and Ugarte (2005), while estimating genetic correlations between conformation traits and milk yield in Churra and Laxta sheep, respectively, calculated correlations that indicated that selection for improved milk yield could have implications on udder morphology. Depth of the udder and placement of the teats were particularly affected, leading to a possible decline in the milking ability of animals in machine-milking environments. Similar findings were observed by Manfredi et al. (2001), indicating that to reduce the decline of milking ability while attempting to improve milk yield, it would be valuable to include conformation traits in breeding programs.

However, while examining the links between conformation traits and milk yield, many of these studies have used cumulated milk yields produced during lactations of different lengths; for example, 120-d milk yield in sheep (Fernandez et al., 1997; Legarra and Ugarte, 2005) or 250-d milk yield in goats (Manfredi et al., 2001; Rupp et al., 2011). With the increased use of random regression models to estimate genetic parameters in goats (Menéndez-Buxadera et al., 2010; Mucha et al., 2014), the opportunity now exists to investigate correlations between conformation traits and milk yield throughout lactation in more detail.

The aims of this study were therefore to (1) estimate genetic parameters for conformation traits in a population of crossbred dairy goats, (2) estimate correlations between all conformation traits, and (3) assess the relationship between conformation traits and milk yield throughout lactation.

\section{MATERIALS AND METHODS}

\section{Conformation Traits}

Conformation trait data were collected from goats based on 2 farms, owned by the same farming business and consisting of related animals, during August and September in 2013 and 2014. The data set comprised data available for 4,220 goats in their first lactation. The traits recorded for each animal, scored by the same recorder, were linear in form and scored using a 9-point scale that was similar to that developed by the French dairy goat breeders' association CAPGENES and used by Manfredi et al. (2001) and Rupp et al. (2011). The data contained information for 3 udder traits, 3 teat traits, and 4 traits relating to legs and feet.

The udder traits, as shown in Figure 1, were as follows: Udder furrow (UF), viewed from the rear of the udder, indicates the prominence of the medial suspensory ligament. A score of 1 indicates that the ligament is highly prominent, with an extreme cleft in the base of the udder, whereas a score of 9 indicates that the ligament is not prominent and there is little or no cleft visible. Udder depth (UD) is the depth of the udder measured compared with the hocks of the animal. Udders scored 1 are close to the ground, whereas those scored 9 are well above the hocks. A score of 5 indicates that the cleft of the udder is at the hock level. Udder attachment (UA) represents the strength of attachment based on the perimeter of the insertion to the abdominal wall. A score of 1 represents udders with a weak and narrow level of attachment, whereas those scored 9 are udders with a large and strong perimeter of attachment.

The teat traits, also shown in Figure 1, were as follows: Teat shape (TS), indicating the diameter and shape of the teat, ranges from wide and conical for score 1 to small and cylindrical for score 9. Teat angle (TA) describes whether the teat, when looking from the side of the animal, is pointing forward (score 1) or toward the rear (score 9); a score of 5 represents teats pointing straight downwards. Teat placement (TP) is scored using a rear view of the udder and gives an indication as to placement of the teats in relation to the medial ligament. Teats pointing outward, away from each other, are scored as 1 , whereas those pointing inward, toward each other, are scored 9; a score of 5 would be given for teats pointing straight down.

The leg and feet traits are shown in Figure 2. The scoring for the front legs is similar to that shown for the back legs. A score of 5 for both the front legs (FL) and back legs (BL) represented legs that were completely straight. Animals with legs that pointed inward at a severe angle, resulting in the hocks $(\mathbf{B L})$ or knees $(\mathbf{F L})$ nearly touching, would be given a score of 1 , whereas the opposite, with the hocks or knees a large distance apart and forming bowed legs, would be scored as a 9 . The front feet (FF) and back feet (BF) scores describe the direction that the hooves are facing when the animal is standing (Figure 2). A score of 1, considered the 


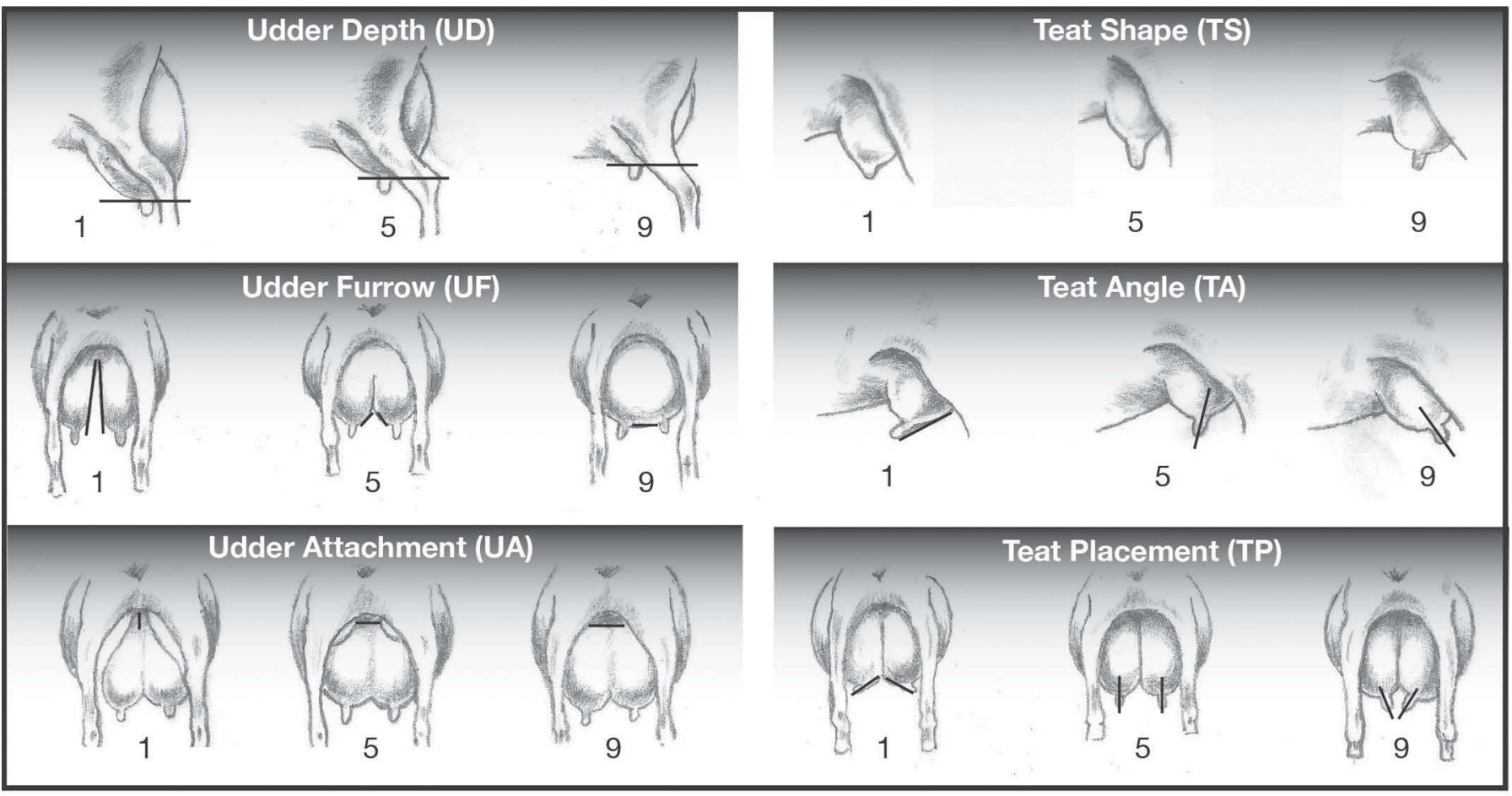

Figure 1. Scoring criteria used for the udder and teat traits.

worst score for both $\mathrm{FF}$ and $\mathrm{BF}$, represented hooves that were facing away from each other. A score of 5 represented hooves pointing outward but at a less severe angle. The best score for $\mathrm{FF}$ and $\mathrm{BF}$ was 9, given to animals with both hooves pointing straight forward. In addition to the conformation traits, test-day milk yield and cumulative milk yield data, up to d 305 , were also available.

\section{Genetic Analysis}

The population of goats used in the study, as described by Mucha et al. (2014), was created using random crossings between 3 breeds: British Alpine, Saanen, and Toggenburg. In each generation, the best performing animals were selected for breeding, leading to the formation of a synthetic breed. No information was available in terms of the breed composition of the animals in the population; therefore, this could not be included in the analyses. The pedigree file used in the analyses contained sire and dam information for a total of 30,139 individuals. Estimates of (co)variance components were obtained by the average information-REML algorithm in the DMU package (Madsen and Jensen, 2008). The model fitted for the conformation traits was

$$
\mathbf{y}=\mathbf{X b}+\mathbf{Z a}+\mathbf{e}
$$

where $\mathbf{y}$ is a vector of observations for the analyzed conformation score; $\mathbf{b}$ is a vector of fixed effects: farm, lactation stage, year of scoring, and birth year; $\mathbf{a}$ is the vector of random additive animal effects; $\mathbf{e}$ is the vector

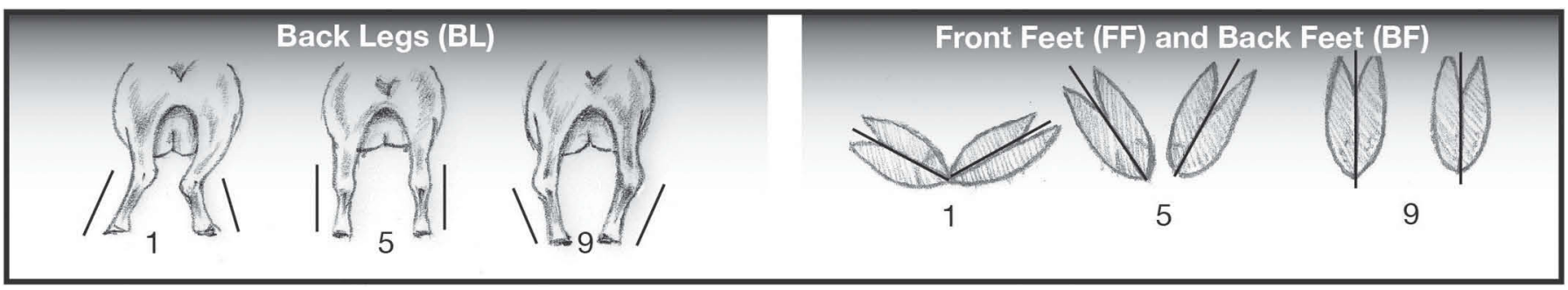

Figure 2. Scoring criteria used for the leg and feet traits. 
of random residual effects, and $\mathbf{X}$ and $\mathbf{Z}$ are incidence matrices relating records to their respective effects. Lactation stage, defined as the number of days between kidding date and scoring date, was grouped into 7 levels to achieve an appropriate distribution: $100 \mathrm{~d}$ or less (939 records), 101-200 d (2,123 records), 201-300 d (536 records), 301-400 d (276 records), 401-500 d (129 records), 501-600 d (114 records), and $601 \mathrm{~d}$ and above (103 records). Year of scoring had 2 levels: 2013 (1,662 records) and 2014 (2,558 records); and birth year had 5 levels: 2009 (49 records), 2010 (162 records), 2011 (661 records), 2012 (1,810 records), and 2013 (1,538 records). Only the direct genetic effect (animal) was fitted in each model because the conformation scores were recorded only once on each individual animal.

Genetic and phenotypic correlations between each individual conformation trait were estimated using a multivariate analysis including all traits. The covariance structure for the multivariate analyses was

$$
\operatorname{Var}\left[\begin{array}{c}
a_{1} \\
a_{2} \\
\vdots \\
a_{10} \\
e_{1} \\
e_{2} \\
\vdots \\
e_{10}
\end{array}\right]=\left[\begin{array}{cccccccc}
\mathbf{A} \sigma_{g 1}^{2} & \mathbf{A} \sigma_{g 1,2} & \cdots & \mathbf{A} \sigma_{g 1,10} & 0 & 0 & 0 & 0 \\
& \mathbf{A} \sigma_{g 2}^{2} & \cdots & \mathbf{A} \sigma_{g 2,10} & 0 & 0 & 0 & 0 \\
& & \cdots & \vdots & \vdots & \vdots & \vdots & \vdots \\
& & & \mathbf{A} \sigma_{g 10}^{2} & 0 & 0 & 0 & 0 \\
& & & & \mathbf{I} \sigma_{e 1}^{2} & \mathbf{I} \sigma_{e 1,2} & \cdots & \mathbf{I} \sigma_{e 1,10} \\
& & & & & \mathbf{I} \sigma_{e 2}^{2} & \cdots & \mathbf{I} \sigma_{e 2,10} \\
& & & & & & \cdots & \vdots \\
& & & & & & & \mathbf{I} \sigma_{e 10}^{2}
\end{array}\right]
$$

where indices 1 and 2 (and so on) indicate the 2 conformation traits, $\mathbf{A}$ is the additive genetic relationship matrix, $\mathbf{I}$ are identity matrices, and $\sigma_{g}^{2}$ and $\sigma_{e}^{2}$ are the genetic and residual variances, respectively.

Milk yield was modeled with a random regression animal model developed in a previous study (Mucha et al., 2014):

$$
\mathbf{y}=\mathbf{X b}+\mathbf{Z a}+\mathbf{W p}+\mathbf{e}
$$

where $\mathbf{y}$ is the vector of test-day observations; $\mathbf{b}$ the vector of fixed effects, consisting of age at kidding, herd-test-day, year-season, and fixed lactation curves modeled by fitting Legendre polynomials (Kirkpatrick et al., 1990) of fourth order; a is a $1 \times 3$ vector of random regression coefficients (Legendre polynomials of second order) for the animal effect; $\mathbf{p}$ is the $1 \times$ 3 vector of random regression coefficients (Legendre polynomials of second order) for the permanent environment effect; and $\mathbf{e}$ is the vector of random residual effect. The matrix $\mathbf{X}$ is the incidence matrix for fixed effects; $\mathbf{Z}$ and $\mathbf{W}$ are matrices of Legendre polynomials of DIM of second order for random animal and permanent environment effect, respectively.

Random effects were assumed normally distributed with zero means and the following covariance structure:

$$
\operatorname{Var}\left[\begin{array}{l}
\mathbf{a} \\
\mathbf{p} \\
\mathbf{e}
\end{array}\right]=\left[\begin{array}{ccc}
\mathbf{A} \otimes \mathbf{G} & 0 & 0 \\
& \mathbf{I} \otimes \mathbf{P} & 0 \\
\text { symm } & & \mathbf{I} \sigma_{e}^{2}
\end{array}\right],
$$

where $\mathbf{G}$ and $\mathbf{P}$ are $3 \times 3$ (co)variance matrices of the random regression coefficients for the animal and permanent environment effects, respectively.

The variance-covariance structures for the bivariate analyses of milk yield and conformation traits were as follows:

$$
\operatorname{Var}\left[\begin{array}{c}
\mathbf{a}_{1} \\
\mathbf{a}_{2} \\
\mathbf{p}_{2} \\
\mathbf{e}_{1} \\
\mathbf{e}_{2}
\end{array}\right]=\left[\begin{array}{ccccc}
\mathbf{A G}_{1} & \mathbf{A G}_{12} & 0 & 0 & 0 \\
& \mathbf{A G}_{2} & 0 & 0 & 0 \\
& & \mathbf{I P}_{2} & 0 & 0 \\
& & & \mathbf{I} \sigma_{e 1}^{2} & \mathbf{I} \sigma_{e 12}^{2} \\
\text { symm } & & & & \mathbf{I} \sigma_{e 2}^{2}
\end{array}\right],
$$

where indices 1 and 2 indicate the 2 traits. The first trait was one of the conformation traits (following model 1), and the second trait was milk yield (following model 2). Matrix $\mathbf{G}_{1}$ contained only one value, $\mathbf{G}_{12}$ contained one row $(1 \times 3)$, whereas $\mathbf{G}_{2}$ and $\mathbf{P}_{2}$ were $3 \times$ 3 (co)variance matrices as defined for model 2. Because the first trait was modeled with model 1 , it had no permanent environment, thus $\mathbf{P}_{1}$ and $\mathbf{P}_{12}$ do not exist.

The genetic covariances between milk yield and conformation traits on the ith DIM were calculated as $\hat{\sigma}_{g i 12}=\mathbf{L}^{\prime}\left(\mathrm{DIM}_{i}\right) \hat{\mathbf{G}}_{12}$, where $\mathbf{L}\left(\mathrm{DIM}_{i}\right)$ is the row vector of Legendre polynomials for $\mathrm{DIM}_{i}$ (Kirkpatrick et al., 1990), of size 3 . The phenotypic covariance was the sum of genetic and residual covariance. An additional analysis with cumulative 305-d milk yield was also performed, using the following model:

$$
\mathbf{y}=\mathbf{X b}+\mathbf{Z a}+\mathbf{e}
$$

where $\mathbf{y}$ is a vector of observations for the $305-\mathrm{d}$ milk yield; $\mathbf{b}$ is a vector of fixed effects: age at kidding, farm, year-season; $\mathbf{a}$ is the vector of random additive animal effects; $\mathbf{e}$ is the vector of random residual effects, and $\mathbf{X}$ and $\mathbf{Z}$ are incidence matrices relating records to their respective effects. Subsequently, bivariate analyses of milk and conformation were performed where conformation traits were modeled using model 1 and 305-d 
milk yield with model 3 . The covariance structure for the bivariate analyses was

$$
\operatorname{Var}\left[\begin{array}{l}
\mathbf{a}_{1} \\
\mathbf{a}_{2} \\
\mathbf{e}_{1} \\
\mathbf{e}_{2}
\end{array}\right]=\left[\begin{array}{cccc}
\mathbf{A} \sigma_{g 1}^{2} & \mathbf{A} \sigma_{g 12} & 0 & 0 \\
& \mathbf{A} \sigma_{g 2}^{2} & 0 & 0 \\
& & \mathbf{I} \sigma_{e 1}^{2} & \mathbf{I} \sigma_{e 12} \\
& & & \mathbf{I} \sigma_{e 2}^{2}
\end{array}\right],
$$

where indices 1 and 2 indicate the 2 traits. The first trait was one of the conformation traits, and the second trait was 305-d milk yield, $\mathbf{A}$ is the additive genetic relationship matrix, I are identity matrices, and $\sigma_{g}^{2}$ and $\sigma_{e}^{2}$ are the genetic and residual variances, respectively.

The standard errors for heritability and genetic and phenotypic correlations were calculated using the methodology proposed by Fischer et al. (2004), with the interpretation proposed by Frigo et al. (2010). Fat and protein contents were not included in the analysis as no data were available from either of the farms contributing data to the study.

\section{RESULTS AND DISCUSSION}

A summary of traits included in the analyses is shown in Table 1. The average scores recorded on both farms were similar for all traits. The front legs trait had the lowest overall standard deviation (0.37), which was a reflection that $88 \%$ of records were a score of 5 . The average daily milk yield during the first lactation was $3.32 \pm 0.003 \mathrm{~kg}$. The additional summary statistics for this trait are provided by Mucha et al. (2014).

\section{Genetic Parameters}

The univariate heritability estimates for each trait, across both farms, ranged from 0.02 to 0.38 (Table 2). The highest estimates were generally associated with the udder- and teat-related traits, whereas those estimated for the legs and feet were lower. The individual traits with the overall highest and lowest heritability estimates were UD (0.38) and FL (0.02), respectively. Manfredi et al. (2001) also observed generally higher estimates for the udder and teat traits compared with the legs and feet. However, although a similar scale and scoring system was used, some of the traits considered by Manfredi et al. (2001) differed from those in the present study. The leg and feet traits similar between both studies were those relating to hock distance (similar to BL in the present study) and feet angle, although no indication was given as to whether this related to the angle of all feet or just the front or back feet. Nonetheless, heritability estimates for hock distance (0.16 and 0.12 for the Alpine and Saanen breeds, respectively) were reasonably similar to those in the present study (0.13). In dairy sheep, de la Fuente et al. (2011) estimated a heritability of 0.18 for rear legs, scored in the same manner but with a slight difference in score definitions. The very low heritability estimate observed for FL is likely due to the lack of variation in scores given for this trait, perhaps influenced by previous selection criteria on the farms, which may have only selected animals with straight front legs to remain in the herd. The heritability of milk yield in this population was 0.56, as estimated by Mucha et al. (2015). The authors suggest that this relatively high estimate could be influenced by the reduction in "noise" due to the automated recording equipment used and the fact that the data originated from just 2 farms.

Considering the udder and teat traits in more detail, the estimates observed in the present study for UD, UF, and TS were in close agreement with those observed by both Manfredi et al. (2001) and Rupp et al. (2011), despite some differences between individual breeds. However, estimates observed for UA (0.15) and TP (0.23) were lower than the values estimated by Manfredi et al. (2001) and Rupp et al. (2011), who both referred to these traits as the rear udder and teat

Table 1. Summary of conformation traits included in the analyses

\begin{tabular}{lcccrc}
\hline Trait & Count $^{1}$ & Minimum & Maximum & Mean & SD \\
\hline Udder furrow (UF) & 4,153 & 2 & 9 & 6.42 & 0.99 \\
Udder depth (UD) & 4,220 & 2 & 9 & 5.93 & 0.94 \\
Udder attachment (UA) & 4,220 & 3 & 9 & 4.69 & 0.68 \\
Teat shape (TS) & 4,198 & 1 & 8 & 4.17 & 1.00 \\
Teat angle (TA) & 4,216 & 1 & 6 & 3.24 & 0.81 \\
Teat placement (TP) & 4,216 & 1 & 8 & 4.88 & 0.81 \\
Front legs (FL) & 4,216 & 2 & 9 & 4.60 & 0.62 \\
Back legs (BL) & 4,219 & 2 & 9 & 8.23 & 0.74 \\
Front feet set (FF) & 4,213 & 3 & 9 & 7.78 & 0.77 \\
Back feet set (BF) & 4,217 & 4 & $3,002.50$ & $1,151.75$ & 325.88 \\
305-d milk yield (kg) & 4,170 & 157.05 & & 9
\end{tabular}

${ }^{1}$ Count reflects the number of animals scored for each trait. Every animal was scored only once for a given trait. 
placement, respectively. The estimates given in those 2 studies ranged from 0.23 to 0.29 for UA and 0.25 to 0.38 for TP. Additionally, the heritability of 0.36 observed for TA was higher than previous estimates, which ranged from 0.15 to 0.22 (Manfredi et al., 2001; Rupp et al., 2011). Compared with the estimates given by Luo et al. (1997) for TP and UD, all of the estimates observed in the current study were low, although some similarities were seen between the UF and TS estimates (referred to as suspensory ligament and teat diameter in the previous study, respectively).

Many of these differences may be related to the number of data records available $(\mathrm{n}=4,220)$ and the breed composition of animals used in this study. The 3 studies discussed above all had data available from just fewer than 19,000 to just over 43,000 animals recorded over several years. It is likely that as more data become available, future estimates may become more accurate, particularly in terms of the standard errors associated with the estimates observed. The lower estimates observed for the feet and leg traits in general could be influenced by farm selection policies already in place, as individuals with undesirable conformation for these traits can become visible from an early age and can be removed before becoming established in the herd. The synthetic nature of the population structure in the current study, based on a history of crossbreeding, also differed from the purebred populations previously referred to. This could also influence the heritability values estimated because of the expected increase in the additive genetic variance in mixed populations compared with purebred populations. Luo et al. (1997) mention data available from crossbred goats, which they refer to as "experimentals," but unfortunately data from these animals were not used in their final analyses to keep consistency with additional data they were using in the study.

\section{Relationships Between Conformation Traits}

The genetic and phenotypic correlations estimated between all of the conformation traits are shown in Table 2. A range of positive and negative genetic correlations were observed, with the highest estimated between UD and UA (0.77) and the lowest between FL and TS and between $\mathrm{BF}$ and $\mathrm{TP}$ (both -0.01). The standard errors associated with the genetic correlations were relatively high, with the highest generally associated with the leg and feet traits. The phenotypic correlations were low to medium, ranging from 0.003 to 0.38 , with standard errors between 0.01 and 0.02 .

Among the udder traits, the genetic correlations ranged from 0.12 to 0.77 , with the highest observed between UD and UA. The relationships between the teat 
traits were similar to those observed among the udder traits, ranging from -0.10 to 0.69 , with negative values estimated between TS and both TA and TP. The moderately high positive correlation between TA and TP (0.69) indicated that selection for improved teat placement would result in improved angle of the teats. The relationships between the udder and teat traits followed a pattern similar to those observed by Manfredi et al. (2001), in both Alpine and Saanen breeds, in terms of direction of the relationships. All were positive, except for those observed between UF and both TA and TP, indicating that as the strength of the medial ligament changed, there was a negative knock-on effect on the angle and placement of the teats.

Genetic correlations estimated between the leg and feet traits ranged from 0.05 to 0.64 . The highest estimate, estimated between $\mathrm{BL}$ and $\mathrm{BF}$ (0.64), suggests that improvements made in back leg conformation would also have a positive effect on the conformation of the back feet. In general, relatively high standard errors were observed between these traits and the other conformation traits, which, in part, are most likely influenced by the low heritability estimates associated with these traits, particularly those relating to FL.

\section{Relationships Between Conformation Traits and Milk Yield Throughout Lactation}

To our knowledge, this is the first time that correlations between conformation traits and milk yield have been estimated throughout lactation in this manner. As many dairy goat herds strive to make improvements in milk yields, it is important to consider the relationship and the effects that this may have on other traits, such as those associated with conformation. The genetic correlations observed between each conformation trait and milk yield during the first lactation are shown in Figure 3. Phenotypic correlations estimated were all close to zero, with the exception of UD, which ranged from -0.35 to -0.07 .

Genetic correlations associated with the udder traits (UF, UD, and UA) are shown in Figure 3a. The correlations estimated between milk yield and UF ranged from -0.42 and 0.18 . Positive values were found up to approximately 50 DIM, after which the values became negative and remained negative throughout the remainder of the lactation. The size of the correlations increased up to around 300 DIM (-0.42), after which they began to steadily fall back toward zero. Standard errors ranged from 0.05 to 0.07 . The correlations observed between milk yield and UD followed a similar pattern; however, unlike UF, all values were negative, ranging from -0.24 and -0.83 . The absolute correlation values increased sharply during the first 100 DIM before continuing to increase, at a slower rate, between 100 and 350 DIM. From 350 DIM onward, the correlations fell back to around -0.27 at 500 DIM. Standard errors were between 0.03 and 0.06 , with the lowest occurring mid lactation between approximately 200 and 300 DIM. Correlation values observed for UA were also all negative, ranging from -0.07 to -0.32 . We detected a gradual increase in the strength of the correlation during the first 70 DIM from a value of -0.21 to -0.32 ,
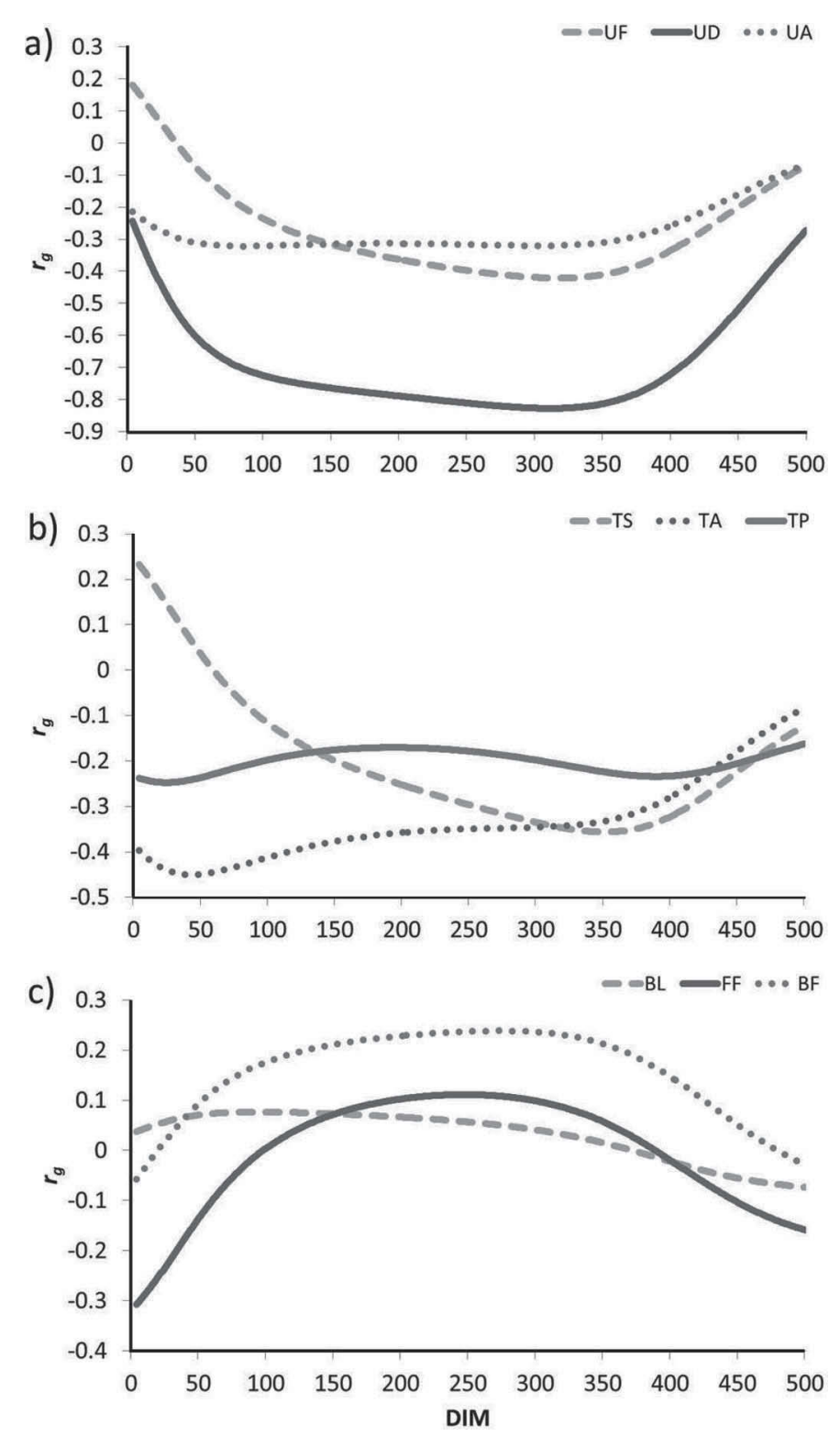

Figure 3. Genetic correlations $\left(\mathrm{r}_{g}\right)$ between milk yield and conformation traits: (a) udder traits - udder furrow (UF), udder depth (UD), and udder attachment (UA); (b) teat traits - teat shape (TS), teat angle (TA), and teat placement (TP); and (c) feet and leg traits - back legs (BL), front feet (FF), and back feet (BF) during 500 d of lactation, based on a random regression model. 
after which values remained around -0.32 until 350 DIM. Standard errors were between 0.06 and 0.09 , with the lowest found during mid lactation. When compared with the genetic correlations estimated with the cumulative milk yield at $305 \mathrm{~d}$, the udder traits behaved consistently, insofar as all correlations observed were negative $(-0.25 \pm 0.13,-0.71 \pm 0.08$, and $-0.28 \pm 0.17$ for UF, UD, and UA respectively). Manfredi et al. (2001), when estimating correlations between udder traits and milk yield at $250 \mathrm{~d}$, also observed negative correlations, ranging from -0.51 to -0.19 , whereas Barillet (2007) comments that, across several different studies in both dairy goats and sheep, correlations between milk yield and UD, although to varying degrees, were always antagonistic. Similar relationships between UD and milk yield have been observed in dairy cattle (Brotherstone, 1994). The correlations that we observed in the current study therefore seem to be in agreement with those estimated previously. Another method of udder scoring, relating to UD, was investigated by Casu et al. (2006), using the degree of suspension of the udder and calculated using the ratio between the udder attachment width and udder height. They suggested this trait as a possible alternative because, in their study, it had a similar heritability as UD, it was highly correlated with UD (0.82), and it had a low and unfavorable genetic correlation with milk yield. In addition, although not investigated in the present study, results reported by Marie-Entacelin et al. (2005) and Rupp et al. (2011) suggest the possibility that selection for improved udder conformation could reduce lactation SCC in sheep and goats, respectively. Overall, the results found in the present study, particularly the highly negative correlations observed between milk yield and UD, suggest that breeding programs would benefit by taking udder traits into account so that selection for productivity is not accompanied by a deterioration in udder conformation, especially in the absence of detailed measures of mastitis.

The genetic correlations associated with the teat traits (TS, TA, and TP) varied throughout the first lactation (Figure $3 \mathrm{~b}$ ). Correlations between milk yield and TS had a similar pattern as the udder traits, in that values became increasingly negative in the early stage of lactation and began to decrease and approach zero toward the end. The values ranged from -0.36 to 0.23 and followed a similar pattern to those observed for UF; standard errors ranged from 0.05 to 0.07 . The correlations estimated between milk yield and both TA and TP followed a different pattern compared with TS, with all estimates remaining negative throughout lactation. During the first 50 DIM, the correlations associated with TA changed from -0.40 to -0.45 . From this point onward, the correlation values began to decrease steadily, levelling off at -0.35 for a period after 250 DIM before falling again after 350 DIM to -0.05 . The standard errors ranged from 0.04 to 0.07 . The correlation values observed for TP ranged from -0.15 to -0.25 and were therefore relatively more stable across lactation compared with the other teat traits. The overall standard errors ranged from 0.05 to 0.08 . The relationship with cumulative milk yield at $305 \mathrm{~d}$ were in general agreement, particularly for TS and TP, with genetic correlations of $-0.35(0.13)$ and $-0.05(0.15)$, respectively. However, the magnitude of the correlation associated with TA $(0.03 \pm 0.13)$, which is essentially zero given the standard error, was different from the estimate from the random regression analyses in early lactation but not very different from the very low negative estimates toward the end of lactation. The relationship between TP and milk yield, as reported by Manfredi et al. (2001), was low, with absolute values below 0.2 , similar to the values observed in the present study between 100 and 300 DIM. Correlations of a similar magnitude between these 2 traits were observed by Fernandez et al. (1997) and Legarra and Ugarte (2005) in different breeds of dairy sheep. Manfredi et al. (2001) reports that there was an extremely weak relationship between TS and milk yield with a correlation of just 0.03 in the Saanen breed, which is very different to the values observed in the present study, which went to -0.36 . Although moderate, the size of the correlations observed for both TS and TA indicate that increased milk yield will affect the size and angle of the teats, which, depending on the milking equipment used, may prove problematic. Rupp et al. (2011) also observed favorable correlations between some teat conformation characteristics and SCC, although this was much more evident in the Saanen breed than in Alpine goats, suggesting that individual breed differences would need to be taken into consideration. The inclusion of teat conformation information, therefore, in addition to the udder traits mentioned previously, would further benefit future breeding programs, particularly in terms of milking ability but possibly also, after further investigation, milk quality and udder health.

The correlations estimated for $\mathrm{BL}, \mathrm{FF}$, and $\mathrm{BF}$ are shown in Figure 3c. The values estimated between milk yield and FL are not shown due to the unreliable nature of the results from the low heritability of FL and the lack of variation in the scores associated with this trait. The correlations associated with BL were associated with high standard errors (0.06-0.10) and not significantly different from zero $(P>0.05)$. The correlations with feet traits both became increasingly positive, at different rates, during the early stages of lactation before beginning to decrease again toward 500 DIM. Both were associated with standard errors ranging from 0.05 
to 0.08 . The FF correlations became more positive during early lactation, from -0.31 at the beginning to 0.11 at 250 DIM. The values then fell again to -0.16 at 500 DIM. Although following a similar pattern to FF, the strength of the correlations observed for BF were higher. Positive correlations were observed between approximately 20 and 480 DIM. The highest correlation was 0.24 at 250 . Like the previous correlations estimated between the conformation traits and milk yield at $305 \mathrm{~d}$, the standard errors were relatively high. The relationship with FF $(0.08 \pm 0.18)$ and $\mathrm{BF}(-0.09 \pm$ $0.15)$ were again both essentially zero. This was similar to the majority of correlations observed throughout mid lactation in the random regression analyses for $\mathrm{FF}$ but only for the correlations associated with BF during early and late lactation. The correlation observed with 305-d milk yield for BL was $0.33( \pm 0.17)$, which was quite different from the low correlations estimated in the random regression analyses (close to zero). This can be interpreted as a reflection of the product of the relative contribution of each daily milk yield to the 305-d yield and the genetic correlations of the trait with daily milk. However, as mentioned previously, the addition of more data records for these traits may improve the reliability of results. In terms of other estimates available in the literature, which are comparable with those presented here, there are very few, due to the investigation of differently defined traits relating to the legs and feet or to the fact that many focus on udder- and teat-related traits. de la Fuente et al. (2011) estimated a small genetic correlation of -0.09 between the back legs and milk yield of Churra ewes, which was markedly different compared with our estimate of 0.33 with milk yield at $305 \mathrm{~d}$.

The results presented improve our knowledge of this population in terms of the conformation traits themselves and their relationship with milk yield. It is unfortunate that no information was available concerning milk composition such as fat, protein, and SCC, as these are also economically important traits to consider. Future studies would therefore benefit from recording this data as well. Additionally, further investigation into the economic values of these traits and the effects of any changes in conformation on aspects such as udder health and longevity of the animals in the herd would be worthwhile. The effect of the different scores will depend on aspects such as the environments in which goats such as these are being milked. For example, the milking machines used may influence which teat and udder scores are the most (and least) desirable. Nonetheless, the heritability and correlation estimates observed for the majority of traits in our study indicate that genetic change can be achieved using conventional quantitative selection methods. In addition, however, the development in genomic selection over recent years provides further opportunities for this population and, indeed, dairy goats worldwide. Although becoming increasingly popular in species such as dairy and beef cattle, genomic selection in dairy goats is still relatively new. A recent study by Mucha et al. (2015), using the same sample population as in this study, found that genomic breeding values could be estimated using a single-step approach. Using such methodology offers potential to identify, at a very early age, animals with favorable conformation characteristics and milk yield, thus improving the efficiency and production of the herd further.

\section{CONCLUSIONS}

Conformation traits investigated in the present study had a low to moderate range of heritabilities, with those relating to the udders and teats providing the highest estimates. Traits relating to the feet and legs were less heritable and less reliable, although this could be improved in the future with the collection of more data records from the population studied. The genetic correlations estimated between the conformation traits and milk yield across the first lactation demonstrate the changes that occur during this period. The majority of the correlations estimated between milk yield and the udder and teat traits were negative; therefore, future breeding programs would benefit from including these traits to ensure that selection for increased productivity is not accompanied by the unwanted deterioration of functional fitness.

\section{ACKNOWLEDGMENTS}

This paper is part of a 3-year project co-funded by the UK innovation agency the Technology Strategy Board (Swindon, UK). The authors gratefully acknowledge cooperation with Angus Wielkopolski and Mark De Hamel from Yorkshire Dairy Goats (Seaton Ross, York, UK) and Marta Rus for her technical assistance in scoring all of the animals used in the study. Many thanks also to David McNeil (SRUC) for his drawings depicting the different conformation scores.

\section{REFERENCES}

Barillet, F. 2007. Genetic improvement for dairy production in sheep and goats. Small Rumin. Res. 70:60-75.

Brotherstone, S. 1994. Genetic and phenotypic correlations between linear type traits and production traits in Holstein-Friesian dairy cattle. Anim. Prod. 59:183-187.

Carta, A., S. Casu, and S. Salaris. 2009. Invited Review: Current state of genetic improvement in dairy sheep. J. Dairy Sci. 92:5814-5833. 
Casu, S., I. Pernazza, and A. Carta. 2006. Feasibility of a linear scoring method of udder morphology for the selection scheme of Sardinian sheep. J. Dairy Sci. 89:2200-2209.

de la Fuente, L. F., G. Fernandez, and F. San Primitivo. 1996. A linear evaluation system for udder traits of dairy ewes. Livest. Prod. Sci. 45:171-178.

de la Fuente, L. F., C. Gonzalo, J. P. Sanchez, R. Rodriguez, J. A. Carriedo, and F. San Primitivo. 2011. Genetic parameters of the linear body conformation traits and genetic correlations with udder traits, milk yield and composition, and somatic cell count in dairy ewes. Can. J. Anim. Sci. 91:585-591.

Fernandez, G., J. A. Baro, L. F. de la Fuente, and F. San Primitivo. 1997. Genetic parameters for linear udder traits of dairy ewes. J. Dairy Sci. 80:601-605.

Fischer, T. M., A. R. Gilmour, and J. H. J. van der Werf. 2004. Computing approximate standard errors for genetic parameters derived from random regression models fitted by average information REML. Genet. Sel. Evol. 36:363-369.

Frigo, E., C. D. Dechow, O. Pedron, and B. G. Cassell. 2010. The genetic relationship of body weight and early-lactation health disorders in two experimental herds. J. Dairy Sci. 93:1184-1192.

Kirkpatrick, M., D. Lofsvold, and M. Bulmer. 1990. Analysis of the inheritance, selection and evolution of growth trajectories. Genetics 124:979-993.

Legarra, A., and E. Ugarte. 2005. Genetic parameters of udder traits, somatic cell score, and milk yield in Latxa sheep. J. Dairy Sci. 88:2238-2245.

Luo, M. F., G. R. Wiggans, and S. M. Hubbard. 1997. Variance component estimation and multitrait genetic evaluation for type traits of dairy goats. J. Dairy Sci. 80:594-600.
Madsen, P., and J. Jensen. 2008. A User's Guide to DMU. University of Aarhus, Tjele, Denmark.

Manfredi, E., A. Piacere, P. Lahaye, and V. Ducrocq. 2001. Genetic parameters of type appraisal in Saanen and Alpine goats. Livest. Prod. Sci. 70:183-189.

Marie-Entacelin, C., J. M. Astruc, D. Porte, H. Larroque, and C. Robert-Granié. 2005. Multiple-trait genetic parameters and genetic evaluation of udder-type traits in Lacaune dairy ewes. Livest. Prod. Sci. 97:211-218.

Menéndez-Buxadera, A., A. Molina, F. Arrebola, M. J. Gil, and J. M. Serradilla. 2010. Random regression analysis of milk yield and milk composition in the first and second lactations of MurcianoGranadina goats. J. Dairy Sci. 93:2718-2726.

Meyer, K., S. Brotherstone, W. G. Hill, and M. R. Edwards. 1987. Inheritance of linear type traits in dairy cattle and correlations with milk production. Anim. Prod. 44:1-10.

Mucha, S., R. Mrode, M. Coffey, and J. Conington. 2014. Estimation of genetic parameters for milk yield across lactations in mixedbreed dairy goats. J. Dairy Sci. 97:2455-2461.

Mucha, S., R. Mrode, M. Coffey, and J. Conington. 2015. Estimation of genomic breeding values for milk yield in UK dairy goats. J. Dairy Sci. 98:8201-8208.

Rupp, R., V. Clément, A. Piacere, C. Robert-Granié, and E. Manfredi. 2011. Genetic parameters for milk somatic cell score and relationship with production and udder type traits in dairy Alpine and Saanen primiparous goats. J. Dairy Sci. 94:3629-3634.

Short, T. H., and T. J. Lawlor. 1992. Genetic parameters of conformation traits, milk yield, and herd life in Holsteins. J. Dairy Sci. 75:1987-1998. 